June 11, 2018

\title{
On the Consistency of the Solutions of the Space Fractional Schrödinger Equation
}

\author{
Selcuk S. Bayin \\ Middle East Technical University \\ Institute of Applied Mathematics \\ Ankara TURKEY 06800
}

\begin{abstract}
Recently it was pointed out that the solutions found in literature for the space fractional Schrödinger equation in a piecewise manner are wrong, except the case with the delta potential. We reanalyze this problem and show that an exact and a proper treatment of the relevant integral proves otherwise. We also discuss effective potential approach and present a free particle solution for the space and time fractional Schrödinger equation in general coordinates in terms of Fox's H-functions.
\end{abstract}

PACS numbers: 03.65.Ca, 02.50.Ey, 02.30.Gp, 03.65.Db 


\section{Introduction}

In a recent article, Jeng et. al. [1] argued that solutions obtained for the space fractional Schrödinger equation in a piecewise fashion are not valid. Their arguments were based on a contradiction that they observed when the ground state wave function of the infinite potential well problem is substituted back into the space fractional Schrödinger equation. They concluded that many exact solutions presented in literature are wrong, except the one with the delta function potential. This not only casts doubt on the existing solutions in literature, but also makes it very difficult to find a meaningful solution. In this manuscript, we show that an exact treatment of the integral that lead them to inconsistency proves otherwise.

Fractional calculus has found to be extremely useful in the study of anomalous diffusion. The fastest way to introduce memory effects into an existing equation is to replace a time derivative with its fractional counterpart. In this regard, recently we have reconsidered the time fractional Schrödinger equation and its separable solutions [2]. The Space fractional Schrödinger equation naturally incorporates non local effects and was first introduced by Laskin [3-7]. It is intriguing that the path integral formulation of quantum mechanics over Lévy paths, which has many interesting examples in nature, leads naturally to the space fractional Schrödinger equation where the space derivative is replaced by the fractional Riesz derivative $[2,7]$. In this regard, Riesz derivative plays a central role in the fractional generalization of the Schrödinger equation, where solutions for various potentials are available [3-9]. It should be emphasized that even thought memory and/or non local effects can be studied via the fractional generalizations of certain derivatives in physical equations, there could also be interesting cases where fractional derivatives alone are not sufficient $[10,11]$. For example, path integral formulations of quantum mechanics over distributions other than the Lévy distribution, would not necessarily lead to a fractional Schrödinger equation. Fractional extension of quantum mechanics that treats coordinates and conjugated momentum equivalently have also been considered in literature [12].

To understand the effects of fractional derivatives in Schrödinger equation, one could try effective potentials within the framework of quantum mechanics. In this regard, we have introduced the effective potential approach for the time fractional Schrödinger equation [2], which allowed us to write the time dependence of the separable solutions, which is a Mittag-Leffler function with an imaginary argument, as the product of its purely oscillating and purely decaying parts. In this manuscript, we also write the effective potential for the space fractional Schrödinger equation and apply it to the infinite well problem. We also present a general solution for the space and time fractional Schrödinger equation in terms of Fox's H-functions in general coordinates. 


\section{Consistency of the Solutions of the Space Fractional Schrödinger Equation}

It is well known that the one dimensional space fractional Schrödinger equation is given as

$$
i \hbar \frac{\partial \Psi}{\partial t}=D_{\alpha}\left(-\hbar^{2} \Delta\right)^{\alpha / 2} \Psi+V \Psi
$$

where $\left(-\hbar^{2} \Delta\right)^{\alpha / 2}$ is the quantum Riesz derivative [7,13]:

$$
\left(-\hbar^{2} \Delta\right)^{\alpha / 2} \Psi(x, t)=\frac{1}{2 \pi \hbar} \int_{-\infty}^{+\infty} d p e^{i p x / \hbar}|p|^{\alpha} \Phi(p, t),
$$

and $\Phi(p, t)$ is the Fourier transform of $\Psi(x, t)$ :

$$
\Phi(p, t)=\int_{-\infty}^{+\infty} d x \Psi(x, t) e^{-i p x / \hbar} .
$$

For the infinite square well potential:

$$
V(x)=\left\{\begin{array}{lll}
0 & \text { if } & |x|<a \\
\infty & \text { if } & |x| \geqslant a
\end{array}\right.
$$

the separable solutions are of the form

$$
\Psi(x, t)=e^{-i E t / \hbar} \psi(x),
$$

where $\psi(x)$ satisfies the eigenvalue problem

$$
D_{\alpha}\left(-\hbar^{2} \Delta\right)^{\alpha / 2} \psi(x)=E \psi(x), \psi(a)=\psi(-a)=0 .
$$

The general solution is obtained as $[4,7,8]$

$$
\psi_{n}(x)=\left\{\begin{array}{cll}
A \cos \left(\frac{n \pi}{2 a}(x+a)\right) & \text { if } & |x|<a \\
0 & \text { if } \quad|x| \geqslant a
\end{array} \quad, n=1,2, \ldots\right.
$$

with the energy eigenvalues

$$
E_{n}=D_{\alpha}\left(\frac{\hbar n \pi}{2 a}\right)^{\alpha} .
$$

To show the inconsistency of these solutions, Jeng et. al. [1] concentrated on the ground state with $n=1$ :

$$
\psi_{1}(x)=\left\{\begin{array}{cll}
A \cos \left(\frac{\pi x}{2 a}\right) & \text { if } & |x|<a \\
0 & \text { if } & |x| \geqslant a
\end{array} .\right.
$$


and argued that this solution, albeit satisfying the boundary conditions, $\psi_{1}(-a)=$ $\psi_{1}(a)=0$, when substituted back into the space fractional Schrödinger equation [Eq. (6)] leads to a contradiction.

The Fourier transform of $\psi_{1}(x)$ can be found as

$$
\phi_{1}(p)=-A \pi\left(\frac{\hbar^{2}}{a}\right) \frac{\cos (a p / \hbar)}{p^{2}-(\pi \hbar / 2 a)^{2}},|x|<a,
$$

which when substituted into Equation (6), gives $\psi_{1}(x)$ as the integral

$$
\psi_{1}(x)=-\frac{A D_{\alpha}}{2 E_{1}}\left(\frac{\hbar}{a}\right) \int_{-\infty}^{+\infty} d p\left(\frac{2 a}{\pi \hbar}\right)^{2} \frac{|p|^{\alpha} \cos (a p / \hbar)}{(2 a p / \pi \hbar)^{2}-1} e^{i p x / \hbar},|x|<a .
$$

Defining a new variable, $q=\frac{2 a}{\pi \hbar} p$, we can also write this as

$$
\psi_{1}(x)=-\frac{A D_{\alpha}}{\pi E_{1}}\left(\frac{\pi \hbar}{2 a}\right)^{\alpha} \int_{-\infty}^{+\infty} d q \frac{|q|^{\alpha} \cos (\pi q / 2)}{q^{2}-1} e^{i \pi q x / 2 a} .
$$

Without evaluating the above integral, Jeng et. al. [1] argued that the right hand side, which they wrote as

$$
\psi_{1}(x)=-\frac{A D_{\alpha}}{\pi E_{1}}\left(\frac{\pi \hbar}{2 a}\right)^{\alpha} 2 \int_{0}^{+\infty} d q \frac{|q|^{\alpha} \cos (\pi q / 2)}{q^{2}-1} \cos (\pi q x / 2 a),
$$

can not satisfy the boundary conditions as $x \rightarrow \pm a$ that $\psi_{1}(x)$ satisfies, thus pointing to an inconsistency. However, an exact evaluation of the integral in Equation (12) proves otherwise. Note that the integral

$$
I=\int_{-\infty}^{+\infty} d q \frac{|q|^{\alpha} \cos (\pi q / 2)}{q^{2}-1} e^{i \pi q x / 2 a}
$$

is a singular integral with poles on the real axis at $q= \pm 1$, which could be evaluated via analytic continuation as a Cauchy principal value integral [13].

Substituting

$$
\cos (\pi q / 2)=\frac{1}{2}\left(e^{i \pi q / 2}+e^{-i \pi q / 2}\right)
$$

we first write $I$ as two integrals:

$$
\begin{aligned}
I & =\frac{1}{2} \int_{-\infty}^{+\infty} d q \frac{|q|^{\alpha} e^{i(x / a+1)(\pi q / 2)}}{(q+1)(q-1)}+\frac{1}{2} \int_{-\infty}^{+\infty} d q \frac{|q|^{\alpha} e^{i(x / a-1)(\pi q / 2)}}{(q+1)(q-1)} \\
& =I_{1}+I_{2}
\end{aligned}
$$

For the first integral, $I_{1}$, we close the contour in the upper half $q$-plane with a semicircular path of radius $R$ and then go around the poles on the real axis in the upper $q$ - plane, with small semicircular paths of radius $\delta$. In the limit as $R \rightarrow \infty$, via Jordan's lemma, the contribution over the large semicircle vanishes, 
thus allowing us to evaluate the value of this integral as a Cauchy principal value in the limit $\delta \rightarrow 0$ as [13]

$$
P V\left(I_{1}\right)=i \pi\left(\frac{i}{2} \cos \frac{x \pi}{2 a}\right) .
$$

Similarly for $I_{2}$, we close the contour this time in the lower $q$-plane and circle around the poles in the lower half $q$-plane to obtain

$$
P V\left(I_{2}\right)=-i \pi\left(-\frac{i}{2} \cos \frac{x \pi}{2 a}\right) .
$$

Adding these we obtain the Cauchy principal value of $I$ as

$$
P V(I)=-\pi \cos \frac{x \pi}{2 a}
$$

which on the contrary to Jeng et. al. [1] vanishes at the boundary as $x \rightarrow \pm a$. When this is substituted back into Equation (12) yields

$$
\psi_{1}(x)=\frac{A D_{\alpha}}{E_{1}}\left(\frac{\pi \hbar}{2 a}\right)^{\alpha} \cos \frac{x \pi}{2 a},|x|<a .
$$

Since the eigenvalue for the ground state is

$$
E_{1}=D_{\alpha}\left(\frac{\pi \hbar}{2 a}\right)^{\alpha}
$$

we again obtain the ground state wave function [Eq. (9)]:

$$
\psi_{1}(x)=A \cos \frac{x \pi}{2 a}, \quad|x|<a,
$$

which satisfies the boundary conditions, hence no inconsistency.

The proof for general $n$ also follows along the same lines. For example, for the odd values of $n$, Equation (12) is replaced by

$$
\psi_{n}(x)=-\frac{A D_{\alpha}}{\pi E_{n}} \sin \left(\frac{n \pi}{2}\right)\left(\frac{n \pi \hbar}{2 a}\right)^{\alpha} \int_{-\infty}^{+\infty} d q \frac{|q|^{\alpha} \cos (n \pi q / 2)}{q^{2}-1} e^{i(n \pi x / 2 a) q} .
$$

Now the Cauchy principal value of the needed integral:

$$
I=\int_{-\infty}^{+\infty} d q \frac{|q|^{\alpha} \cos (n \pi q / 2)}{q^{2}-1} e^{i(n \pi x / 2 a) q}
$$

is found as

$$
P V(I)=-\pi \sin \left(\frac{n \pi}{2}\right) \cos \left(\frac{n \pi x}{2 a}\right),
$$

which when substituted back into Equation (24) yields

$$
\begin{aligned}
\psi_{n}(x) & =\frac{A D_{\alpha}}{\pi E_{n}} \sin ^{2}\left(\frac{n \pi}{2}\right)\left(\frac{n \pi \hbar}{2 a}\right)^{\alpha} \cos \left(\frac{n \pi x}{2 a}\right), \\
& =A \cos \left(\frac{n \pi x}{2 a}\right), n=1,3,5, \ldots
\end{aligned}
$$

Again, no contradiction. The proof for the even values of $n$ is similar. 


\section{Effective Potential for the Space Fractional Schrödinger Equation}

For the separable solutions of the free space fractional Schrödinger equation we use Laskin's notation:

$$
i \hbar \frac{\partial}{\partial t} \Psi(x, t)=-D_{\beta}(\hbar \nabla)^{\beta} \Psi(x, t),
$$

where $\nabla^{\beta}$ is the Riesz derivative and

$$
\left(-\hbar^{2} \Delta\right)^{\alpha / 2} \Psi(x, t)=-(\hbar \nabla)^{\beta} \Psi(x, t) .
$$

For a separable solution, $\Psi(x, t)=T(t) X(x)$, found by solving the free space fractional Schrödinger equation we can define an effective potential in terms of the Schrödinger equation as

$$
V_{\text {eff. }}(x)=\frac{\hbar^{2}}{2 m} \frac{1}{X(x)}\left(\frac{d^{2} X(x)}{d x^{2}}\right)-\frac{D_{\beta}(\hbar \nabla)^{\beta} X(x)}{X(x)} .
$$

For separable solutions, the free space fractional Schrödinger equation reduces to

$$
\begin{aligned}
i \hbar \frac{d T(t)}{d t} & =E_{n} T(t), \\
-D_{\beta}(\hbar \nabla)^{\beta} X(x) & =E_{n} X(x),
\end{aligned}
$$

where the energy eigenvalues, $E_{n}$, come from the solution of Equation (33) with the appropriate boundary conditions and, the time dependence is given as

$$
T(t)=e^{-i\left(E_{n} / \hbar\right) t} .
$$

Since in the presence of a potential, $V(x)$, the corresponding fractional Hamiltonian:

$$
H_{\beta}=-D_{\beta}(\hbar \nabla)^{\beta}+V(x),
$$

is hermitian, the energy eigenvalues are real [7]. Thus, we can also write

$$
V_{e f f .}(x)=\frac{\hbar^{2}}{2 m} \frac{1}{X(x)}\left(\frac{d^{2} X(x)}{d x^{2}}\right)+E_{n} .
$$

The equivalent Schrödinger equations are now written as

$$
\begin{aligned}
i \hbar \frac{d T(t)}{d t} & =E_{n} T(t), \\
-\frac{\hbar^{2}}{2 m} \frac{1}{X(x)}\left(\frac{d^{2} X(x)}{d x^{2}}\right)+V_{\text {eff. }}(x) & =E_{n},
\end{aligned}
$$

which are to be solved with the same boundary conditions. 


\section{A. Effective Potential for the Infinite Well}

For the box problem, using the normalization condition

$$
\int_{-a}^{a} d x|\Psi(x, t)|^{2}=1
$$

the complete wave function is given as

$\Psi(x, t)=\left\{\begin{array}{ll}\frac{1}{\sqrt{a}} e^{-(i / \hbar) E_{n} t} \sin \frac{n \pi}{2 a}(x+a) & |x|<a \\ 0 & , \quad|x| \geq a\end{array} \quad, n=1,2, \ldots\right.$,

where

$$
E_{n}=D_{\beta}\left(\frac{n \pi \hbar}{2 a}\right)^{\beta} \text { and } \Psi(-a, t)=\Psi(a, t)=0 .
$$

The effective potential is now written as

$$
V_{\text {eff. }}(x)=D_{\beta}\left(\frac{n \pi \hbar}{2 a}\right)^{\beta}-\frac{\hbar^{2}}{2 m}\left(\frac{n \pi}{2 a}\right)^{2} .
$$

In other words, the effective potential shifts the energy levels by a constant as a function of the quantum number $n$.

\section{Free Particle Solution of the Space and Time Frac- tional Schrödinger Equation}

We now consider the free Schrödinger equation with both time and space fractional derivatives [2]:

$$
\frac{\partial^{\alpha}}{\partial t^{\alpha}} \Psi(x, t)=\frac{i^{\alpha}}{\hbar} \check{D}_{\alpha, \beta}(\hbar \nabla)^{\beta} \Psi(x, t), 0<\alpha<1,1<\beta<2,
$$

where $\frac{\partial^{\alpha}}{\partial t^{\alpha}}$ is the Caputo derivative (Appendix A). Performing a Wick rotation gives the Bloch equation:

$$
\frac{\partial^{\alpha}}{\partial t^{\alpha}} \Psi(x, t)=\frac{1}{\hbar} \check{D}_{\alpha, \beta} \hbar^{\beta} \nabla_{x}^{\beta} \Psi(x, t), 0<\alpha<1,1<\beta<2,
$$

where $\check{D}_{1,2}=1 / 2 m$. Using the boundary conditions

$$
\Psi(x, 0)=\delta(x) \text { and } \lim _{x \rightarrow \pm \infty} \Psi(x, t) \rightarrow 0,
$$

we take the Laplace transform with respect to time and the Fourier transform with respect to space to obtain the Fourier-Laplace transform of the solution 
[14]. Finding the inverse transform and then performing an inverse Wick rotation, yields the wave function in integral form as

$$
\Psi(x, t)=\frac{\Psi_{0}}{2 \pi} \int_{-\infty}^{+\infty} e^{-i k x} E_{\alpha}\left(-\frac{i^{\alpha}}{\hbar} \check{D}_{\alpha, \beta} \hbar^{\beta} k^{\beta} t^{\alpha}\right) d k,
$$

where $E_{\alpha}(z)$ is the Mittag-Leffler function [2]. We can also write $\Psi(x, t)$ as

$$
\Psi(x, t)=\frac{\Psi_{0}}{\pi} \int_{0}^{+\infty} \cos k x E_{\alpha}\left(-\frac{i^{\alpha}}{\hbar} \check{D}_{\alpha, \beta} \hbar^{\beta} k^{\beta} t^{\alpha}\right) d k .
$$

This wave function satisfies both the time and the space fractional Schrödinger equation [Eq. (43)]

In terms of $H$-functions (Appendix B) Equation (47) can be written as

$$
\Psi(x, t)=\frac{\Psi_{0}}{\pi} \int_{0}^{+\infty} \cos k x H_{1,2}^{1,1}\left(\left.\frac{i^{\alpha}}{\hbar} \check{D}_{\alpha, \beta} \hbar^{\beta} k^{\beta} t^{\alpha}\right|_{(0,1),(0, \alpha)} ^{(0,1)}\right) d k
$$

which can be integrated by using the properties of the $\mathrm{H}$-functions [15] as

$$
\Psi(x, t)=\frac{\Psi_{0}}{\sqrt{\pi}|x|} H_{3,2}^{1,2}\left(\left.\frac{i^{\alpha}}{\hbar} \check{D}_{\alpha, \beta} \hbar^{\beta} t^{\alpha}\left(\frac{2}{|x|}\right)^{\beta}\right|_{(0,1),(0, \alpha)} ^{(1 / 2, \beta / 2),(0,1),(0, \beta / 2)}\right) .
$$

To determine $\Psi_{0}$ the final wave function has to be normalized as

$$
\int|\Psi(x, 0)|^{2} d x=1 .
$$

Case I:

For $\beta=2$, this solution becomes the free particle solution of the time fractional Schrödinger equation as

$$
\Psi(x, t)=\frac{\Psi_{0}}{\sqrt{\pi}|x|} H_{3,2}^{1,2}\left(\left.\frac{4 i^{\alpha} D_{\alpha} t^{\alpha}}{|x|^{2}}\right|_{(0,1),(0, \alpha)} ^{(1 / 2,1),(0,1),(0,1)}\right), D_{\alpha}=\check{D}_{\alpha, 2} \hbar .
$$

This can also be shown to be equal to

$$
\Psi(x, t)=\frac{\Psi_{0}}{\sqrt{\pi}|x|} H_{1,2}^{2,0}\left(\left.\frac{|x|^{2}}{4 i^{\alpha} D_{\alpha} t^{\alpha}}\right|_{(1 / 2,1),(1,1)} ^{(1, \alpha)}\right)
$$

or to

$$
\Psi(x, t)=\frac{\Psi_{0}}{|x|} H_{1,1}^{1,0}\left(\left.\frac{|x|^{2}}{i^{\alpha} D_{\alpha} t^{\alpha}}\right|_{(1,2)} ^{(1, \alpha)}\right) .
$$


In the limit as $\alpha \rightarrow 1$, this becomes

$$
\Psi(x, t)=\frac{\Psi_{0}}{\left(4 \pi i D_{1} t\right)^{1 / 2}} \exp \left(-\frac{|x|^{2}}{4 i D_{1} t}\right),
$$

where $D_{1}=\hbar / 2 m$.

Case II:

When $\alpha=1$, we obtain the wave function as

$$
\Psi(x, t)=\frac{\Psi_{0}}{\sqrt{\pi}|x|} H_{3,2}^{1,2}\left(\left.\frac{i}{\hbar} D_{\beta} \hbar^{\beta} t\left(\frac{2}{|x|}\right)^{\beta}\right|_{(0,1),(0,1)} ^{(1 / 2, \beta / 2),(0,1),(0, \beta / 2)}\right),
$$

which satisfies

$$
i \hbar \frac{\partial}{\partial t} \Psi(x, t)=-D_{\beta} \hbar^{\beta} R_{x}^{\beta} \Psi(x, t), 1<\beta<2,
$$

where

$$
\check{D}_{1, \beta}=D_{\beta} .
$$

This solution was also given by $[8]$ in the form:

$$
\Psi(x, t)=\frac{\pi \Psi_{0}}{\beta|x|} H_{2,2}^{1,1}\left(\left.\frac{1}{\hbar}\left(\frac{\hbar}{i D_{\beta} t}\right)^{1 / \beta}|x|\right|_{(1,1),(1,1 / 2)} ^{(1,1 / \beta),(1,1 / 2)}\right) .
$$

\section{Conclusions}

Successful applications of fractional calculus to anomalous diffusion attracted researchers from many different branches of science and engineering into this intriguing branch of mathematics [15-19]. Applications of fractional calculus usually starts by replacing certain derivatives in the evolution or the transport equations with their fractional counterparts. In general, replacing a time derivative with its fractional counterpart incorporates memory effects into the system and makes the process non Markovian, while a replacement of a space derivative introduces global or non local effects.

In 2000, Laskin [3-7] introduced the path integral formulation of quantum mechanics over Lévy paths and showed that the corresponding equation of motion is the space fractional Schrödinger equation. For the infinite potential well problem, the complete wave function is given as $[7,8]$

$$
\Psi(x, t)=\left\{\begin{array}{ll}
\frac{e^{-(i / \hbar) E_{n} t}}{\sqrt{a}} \sin \frac{n \pi}{2 a}(x+a) & , \quad|x|<a \\
0 & , \quad|x| \geq a
\end{array} \quad, n=1,2, \ldots .\right.
$$



Since the energy operator is now given as $i \hbar \frac{d}{d t}$, the energy eigenvalues are given
as

$$
E_{n}=D_{\beta}\left(\frac{\pi n \hbar}{2 a}\right)^{\beta}, n=1,2, \ldots
$$

Note that for the space fractional Schrödinger equation, the Hamiltonian, $H=$ $-D_{\beta}\left(\hbar \frac{\partial}{\partial x}\right)^{\beta}+V(x)$, is hermitian. An alternate approach to space fractional Schrödinger equation is given by Herrmann [12].

It is true that the Riesz derivative requires a knowledge of the wave function over the entire space. For the infinite well problem, the system is confined to the region $|x| \leq a$ with $\Psi(x, t)=0$ for $|x| \geq a$. Since the solution for $|x|<a$, also satisfies the boundary conditions as $x \rightarrow \pm a$, the solution inside the well is consistent with the outside. In this regard, it is not true that the above solution ignores non locality and is valid only for $\beta \approx 1$ [1,12 pg. 107].

In general, in the presence of a potential, $V(x)$, the time independent space fractional Schrödinger equation is given as

$$
D_{\alpha}\left(-\hbar^{2} \Delta\right)^{\alpha / 2} \psi(x ; E, \alpha)+V(x) \psi(x ; E, \alpha)=E \psi(x ; E, \alpha),
$$

which is to be solved with the appropriate boundary conditions. The Fourier transform of Equation (61) yields a dispersion relation of the form $\phi(p ; E, \alpha)$, where the wave function is given as

$$
\psi(x ; E, \alpha)=\frac{1}{2 \pi \hbar} \int_{-\infty}^{+\infty} d p \phi(p ; E, \alpha) e^{i p x / \hbar} .
$$

Since quantization follows from the boundary conditions that $\psi(x ; E, \alpha)$ satisfies, granted that the above integral exists, solutions satisfying the given boundary conditions can not lead to an inconsistency [7-9].

After showing the consistency of the solutions of the space fractional Schrödinger equation for the one dimensional infinite square well, we have also given the effective potential for the space fractional Schrödinger equation. This is the potential that the Schrödinger equation with the same boundary conditions will yield the same wave function as the fractional case. For the space and time fractional Schrödinger equation, we give the free particle solution in terms of $H$-functions, which in the appropriate limits reproduces the previous solutions. The solutions we give for the free particle are in general coordinates. 


\section{APPENDIX}

\section{Appendix A. Basic Definitions of the Fractional Deriva- tives and Integrals}

The Caputo definition of fractional derivative is given as

$$
\left[\frac{d^{q} f(t)}{d t^{q}}\right]_{C}=\frac{1}{\Gamma(1-q)} \int_{0}^{t}\left(\frac{d f(\tau)}{d \tau}\right) \frac{d \tau}{(t-\tau)^{q}}, 0<q<1,
$$

Laplace transform of the Caputo derivative is

$$
£\left\{{ }_{0}^{C} \mathbf{D}_{t}^{q} f(t)\right\}=s^{q} \widetilde{f}(s)-\left.\sum_{k=0}^{n-1} s^{q-k-1} \frac{d^{k} f(t)}{d t^{k}}\right|_{t=0}, n-1<q \leq n,
$$

where ${ }_{0} \mathbf{D}_{t}^{q} f(t) \equiv \frac{d^{q} f}{d t^{q}}$.

In writing the space fractional diffusion equation or the space fractional Schrödinger equation, we use the Riesz derivative which is defined with respect to its Fourier transform:

$$
\mathcal{F}\left\{\mathbf{R}_{x}^{q} f(x)\right\}=-|\omega|^{q} g(\omega), 0<q<2 .
$$

This yields the Riesz derivative as

$$
\mathbf{R}_{x}^{q} f(x)=-\frac{1}{2 \pi} \int_{-\infty}^{+\infty}|\omega|^{q} g(\omega) e^{i \omega x} d \omega
$$

where $g(\omega)$ is the Fourier transform of $f(x)$. Note that

$$
\mathbf{R}_{x}^{2} f(x)=\frac{d^{2}}{d x^{2}} f(x)
$$

\section{Appendix B. Fox's H-Function}

In 1961 Fox introduced the $H$-function, which gives a general way of expressing a wide class of functions encountered in applied mathematics. $H$-function provides an elegant and an efficient formalism to handle problems in fractional calculus. Fox's $H$-function is a generalization of the Meijer's $G$-function and is defined with respect to a Mellin-Barnes type integral [15]:

$$
\begin{aligned}
H_{p, q}^{m, n}(z) & =H_{p, q}^{m, n}\left(\left.z\right|_{\left(b_{q}, B_{q}\right)} ^{\left(a_{p}, A_{p}\right)}\right)=H_{p, q}^{m, n}\left(\left.z\right|_{\left(b_{1}, B_{1}\right), \ldots,\left(b_{q}, B_{q}\right)} ^{\left(a_{1}, A_{1}\right), \ldots,\left(a_{p}, A_{p}\right)}\right) \\
& =\frac{1}{2 \pi i} \int_{C} h(s) z^{-s} d s,
\end{aligned}
$$


where

$$
h(s)=\frac{\prod_{j=1}^{m} \Gamma\left(b_{j}+B_{j} s\right) \prod_{j=1}^{n} \Gamma\left(1-a_{j}-A_{j} s\right)}{\prod_{j=n+1}^{p} \Gamma\left(a_{j}+A_{j} s\right) \prod_{j=m+1}^{q} \Gamma\left(1-b_{j}-B_{j} s\right)},
$$

$m, n, p, q$ are positive integers satisfying $0 \leq n \leq p, 1 \leq m \leq q$, and empty products are taken as unity. Also, $A_{j}, j=1, \ldots, p$, and $B_{j}, j=1, \ldots, q$, are real positive numbers, and $a_{j}, j=1, \ldots, p$, and $b_{j}, j=1, \ldots, q$, are in general complex numbers satisfying

$$
A_{j}\left(b_{h}+\nu\right) \neq B_{h}\left(a_{j}-\lambda-1\right) \text { for } \nu, \lambda=0,1, \ldots ; h=1, \ldots, m, j=1, \ldots, n .
$$

The contour $C$ is such that the poles of $\Gamma\left(b_{j}+B_{j} s\right), j=1, \ldots, m$, are separated from the poles of $\Gamma\left(1-a_{j}-A_{j} s\right), j=1, \ldots, n$ such that the poles of $\Gamma\left(b_{j}+B_{j} s\right)$ lie to the left of $C$, while the poles of $\Gamma\left(1-a_{j}-A_{j} s\right)$ are to the right of $C$. The poles of the integrand are assumed to be simple. The $H$-function is an analytic function of $z$ for every $|z| \neq 0$ when $\mu>0$ and for $0<|z|<1 / \beta$ when $\mu=0$, where $\mu$ and $\beta$ are defined as

$$
\mu=\sum_{j=1}^{q} B_{j}-\sum_{j=1}^{p} A_{j}
$$

and

$$
\beta=\prod_{j=1}^{p} A_{j}^{A_{j}} \prod_{j=1}^{q} B_{j}^{-B_{j}}
$$

Fox's $H$-function is very useful in the study of stochastic processes and in solving fractional diffusion equations (Glöckle and Nonnenmacher [20], West and Grigolini [21]). For example, the following useful formula for the RiemannLiouville fractional derivative of the $H$-function:

$$
{ }_{0}^{R-L} \mathbf{D}_{z}^{\beta}\left[z^{a} H_{p, q}^{m, n}\left(\left.(c z)^{b}\right|_{\left(b_{j}, B_{j}\right)} ^{\left(a_{j}, A_{j}\right)}\right)\right]=z^{a-\beta} H_{p+1, q+1}^{m, n+1}\left(\left.(c z)^{b}\right|_{\left(b_{j}, B_{j}\right),(\beta-a, b)} ^{(-a, b),\left(a_{j}, A_{j}\right)}\right),
$$

where $a, b>0$ and $a+b \min \left(b_{j} / B_{j}\right)>-1,1 \leq j \leq m$, can be used to find solutions to the fractional diffusion equation by tuning the indices to appropriate values. Similarly, the Laplace transform of the $H$-function can be obtained by using the formula [15]

$$
£\left\{x^{\rho-1} H_{p, q+1}^{m, n}\left(\left.a x^{\sigma}\right|_{\left(b_{q}, B_{q}\right),(1-\rho, \sigma)} ^{\left(a_{p}, A_{p}\right)}\right)\right\}=s^{-\rho} H_{p, q}^{m, n}\left(\left.a s^{-\sigma}\right|_{\left(b_{q}, B_{q}\right)} ^{\left(a_{p}, A_{p}\right)}\right),
$$

where the inverse transform is given as

$$
£^{-1}\left\{s^{-\rho} H_{p, q}^{m, n}\left(\left.a s^{\sigma}\right|_{\left(b_{q}, B_{q}\right)} ^{\left(a_{p}, A_{p}\right)}\right)\right\}=x^{\rho-1} H_{p+1, q}^{m, n}\left(\left.a x^{-\sigma}\right|_{\left(b_{q}, B_{q}\right)} ^{\left(a_{p}, A_{p}\right),(\rho, \sigma)}\right),
$$

where

$$
\rho, \alpha, s \in \mathbb{C}, \operatorname{Re}(s)>0, \sigma>0
$$

and

$$
\operatorname{Re}(\rho)+\sigma \max _{1 \leq i \leq n}\left[\frac{1}{A_{i}}+\frac{\operatorname{Re}\left(a_{i}\right)}{A_{i}}\right]>0,|\arg a|<\frac{\pi \theta}{2}, \theta=\alpha-\sigma .
$$


Acknowledgement 1 I would like to thank Jean Krisch and Nick Laskin for valuable comments. 


\section{References}

${ }^{1}$ M. Jeng, S.-L.-Y. Xu, E. Hawkins and J. M. Schwarz, J. Math. Phys. 51, $062102(2010)$.

${ }^{2}$ S. S. Bayin, arXiv:1103.3295v3 [math-ph] (2011).

${ }^{3}$ N. Laskin, Phys. Rev. E 62, 3135 (2000).

${ }^{4}$ N. Laskin, Chaos 10, 780 (2000).

${ }^{5}$ N. Laskin, Phys. Rev. E 66, 056108 (2002).

${ }^{6}$ N. Laskin, arXiv:quad-ph/0504106r1 (2005).

${ }^{7}$ N. Laskin, arXiv:1009.5533v1 [math-ph] (2010).

${ }^{8}$ X. Guo and M. Xu, J. Math. Phys. 47, 082104 (2006).

${ }^{9}$ J. Dong and M. Xu, J. Math. Phys. 48, 072105 (2007).

${ }^{10}$ A. Iomin, arXiv:math-ph/0909.1183v1 (2009).

${ }^{11}$ A. Iomin, arXiv:quant-ph/1108.6178v1 (2011).

${ }^{12}$ R. Herrmann, Fractional Calculus (World Scientific, 2011).

${ }^{13}$ S. S. Bayin, Mathematical Methods in Science and Engineering (Supplements of Ch 14, Wiley, 2006).

${ }^{14}$ A. M. A. El-Sayed and M. Gaber, EJTP 3, 81 (2006).

${ }^{15}$ A. M. Mathai, R. S. Saxena and H. J. Haubold The H-Function, Theory and Application (Springer, 2010).

${ }^{16}$ K. B. Oldham and J. Spanier, The Fractional Calculus (Dover, 1974).

${ }^{17}$ I. Podlubny, Fractional Differential Equations (Academic Press, 1999).

${ }^{18}$ R. Hilfer (editor), Fractional Calculus, Applications in Physics (World Scientific, 2000).

${ }^{19}$ A. A. Kilbas, H. M. Srivastava and J. J. Trujillo, Theory and Applications of Fractional Differential Equations (Elsevier, 2006).

${ }^{20}$ W. G. Glöckle and F. Nonnenmacher, J. Stat. Phys. 71, 741 (1993).

${ }^{21}$ B. J. West and P. Grigolini, Phys. Rev. E 55, 99 (1997). 